\title{
Implicancias pedagógicas y administrativas de la evaluación cualitativa con expresión de calificaciones en letras Caso Universidad Nacional del Centro del Perú
}

\author{
Filomeno Tarazona Pérez \\ Universidad Nacional del Centro del Perú \\ ftarazona@uncp.edu.pe • https://orcid.org/0000-0003-2858-7467 \\ Isabel Margarita Aliaga Contreras \\ Universidad Nacional del Centro del Perú \\ ialiaga@uncp.edu.pe • https://orcid.org/0000-0002-4527-9880
}

\section{Resumen}

Es una investigación exploratoria de prognosis con enfoque cualitativo. Su propósito es, explorar opiniones sobre posibles implicancias pedagógicas y administrativas de la Evaluación Cualitativa con calificativos en letras, en el hipotético caso, se implementara en la UNCP. En la recopilación de datos, se ha ensayado la técnica de Focus Group o grupos focales. Se organizó y ejecutó cuatro grupos focales en la estructura académica y administrativa de la UNCP: Alta Dirección, Decanos de facultades, Directores académicos, Docentes expertos que desarrollan asignaturas de evaluación. Los resultados muestran que, no existe necesidad prioritaria pedagógica ni administrativa para implementar el uso de la evaluación cualitativa con expresión de calificativos en letras; asimismo, los entrevistados muestran informaciones elementales, superficiales y ambiguas sobre la evaluación cualitativa, tanto en sus conceptos, los procesos operativos así como instrumentales. En consecuencia, la probabilidad de la aplicación de evaluaciones cualitativas con expresión de calificativos en letras en la UNCP, no suscita expectativas como temas académicos ni administrativos. La información predominante en materia de evaluación es cuantitativa en el modelo positivista de Variable, indicadores, reactivos, respuestas y notas con procesamiento estadístico básico. Las nociones de evaluación en administración es fundamentalmente de expresión numérica.

Palabras clave: Evaluación, Implicancias pedagógicas, implicancias administrativas, Calificación en letras.

\footnotetext{
Abstract

It is an exploratory prognosis investigation with a qualitative approach. Its purpose is to explore opinions about possible pedagogical and administrative implications of the Qualitative Evaluation with qualifiers in letters, in the hypothetical case, it will be implemented in the UNCP. In data collection, the Focus Group technique or focus groups has been tried. Four focus groups were organized and executed in the academic and administrative structure of the UNCP: senior management, dean of faculties, academic directors and expert teachers abaout evaluation. The results show that there is no pedagogical or administrative priority need to implement the use of qualitative evaluation with expression of qualifiers in letters; Likewise, the interviewees show elementary, superficial and ambiguous informa-
} 
tion on qualitative evaluation, both in terms of its concepts, operational processes as well as instrumental ones. Consequently, the probability of the application of qualitative evaluations with the expression of qualifiers in letters at the UNCP does not raise expectations as academic or administrative issues. The predominant information regarding evaluation is quantitative in the positivist model of variable, indicators, reagents, answers and notes with basic statistical processing. The notions of evaluation in administration is fundamentally of numerical expression.

Keywords: Evaluation, Pedagogical implications, administrative implications, Letter grade.

\section{Introducción}

La universidad es la institución educativa del más alto nivel educativo de la sociedad. Está dedicada fundamentalmente a la docencia, la investigación científica, la extensión cultural y proyección social, así como la responsabilidad social universitaria. Está definida como “...una comunidad académica orientada a la investigación y a la docencia, que brinda una formación humanista, cientifica y tecnológica con una clara conciencia de nuestro país como realidad multicultural. Adopta el concepto de educación como derecho fundamental y servicio público esencial. Está integrada por docentes, estudiantes y graduados” (Ley Universitaria 30220), como tal, es la entidad que debe orientar la opinión científica y técnica de comunidad.

De ahí, la universidad como institución formadora de profesionales, evalúa todas sus funciones misionales: la enseñanza para la formación profesional, la investigación científica disciplinaria de su carrera, la extensión cultural y proyección social, la Responsabilidad Social Universitaria de la conservación, difusión y la democratización de la cultura. Además, la universidad evalúa todos los procesos, productos y soportes institucionales como son los docentes, la infraestructura operativa y de servicios.

En el contexto conceptual y misional de la universidad arriba expuesto, partimos del principio "de que la evaluación forma parte del currículo universitario. Es decir, forma parte del proyecto formativo que cada Facultad desarrolla. La formación que la Universidad ofrece posee algunas características particulares que la diferencian de la formación que se ofrece en otros centros formativos. La principal de es su carácter netamente profesionalizador y de "acreditación". (Amparo Fernández Marcha: Instituto de Ciencias de la Educación Universidad Politécnica de Valencia; p.3) En ese sentido, la Universidad garantiza que los estudiantes que superan los estudios y completan su formación o cuando menos alcanzan el nivel suficiente, es después de haber sido sometidos a evaluaciones y están preparados para ejercer la profesión que han seguido como carrera profesional.

La función formadora de profesionales mediante la enseñanza universitaria del docente, pedagogo o no, planifica, implementa, ejecuta y evalúa el desarrollo de las capacidades, conocimientos, valores y actitudes de los estudiantes en una determinada disciplina científica y técnica de un programa de estudios. En esta función, en la actualidad, la evaluación se realiza tanto del aprendizaje conceptual o teórico, procedimental o metodológico y el actitudinal o de comportamiento individual y social, en la dirección del perfil de egreso previamente planificado en los diseños curriculares. La evaluación actual en las universidades, es esencialmente y oficialmente cuantitativo para los efectos de control de productos académicos, cuyos califica- 
tivos tienen expresión numérica con procesamiento matemático - estadísticos. En esta área los distintos períodos y procesos de la evaluación como de entrada o diagnóstica, la de proceso o formativa y de salida o sumativa que se considera verificadora de logros en las competencias del área formativa, tienen expresión cuantitativa, aunque pueden adoptarse sus equivalentes cualitativos basados en determinados rangos vigesimales o centesimales. Asimismo, las actas promocionales que constituyen, informe de resultados del proceso formativo semestral tienen expresión cuantitativa, así como los promedios ponderados son fórmulas matemáticas (Art. $124^{\circ}$ Reglamento Académico General UNCP). Del mismo modo, a la finalización del semestre académico se reporta el orden de méritos de los estudiantes teniendo en cuenta el promedio ponderado que es también una expresión cuantitativa; igual sucede con la determinación de los quinto, tercio y décimo superior con operaciones matemáticas y estadísticas.

La evaluación de los aprendizajes de investigación y la producción de conocimientos es un proceso por el cual los docentes, estudiantes, egresados y trabajadores profesionales producen conocimientos nuevos conocimientos en sus correspondientes disciplinas. La evaluación en este rubro también se realiza con calificativos numéricos en intervalos, tanto del Plan o Proyecto de Investigación, la evaluación del Informe de investigación, así como las exposiciones de sustentación de tesis como resultados de la investigación tienen expresión numérica de intervalos. Con los mismos procedimientos se realizan también la evaluación de la Extensión Cultural y Proyección Social, la Responsabilidad Social Universitaria y otros procesos y productos de la actividad universitaria.

Por otro lado, la Universidad, también evalúa el desempeño docente desde su postulación, admisión y desempeño profesional mediante reglamentos que tienen estructura estadística con tabla de puntuaciones; de la misma manera el monitoreo del desempeño docente y la encuesta de apreciación estudiantil sobre el trabajo docente, además de la evaluación para la ratificación, ascensos y promociones, tienen procedimientos matemático estadísticos.

Sin embargo, es también muy notable en las universidades peruanas, la predominancia del empirismo de la evaluación. Existe escasa preocupación por la evaluación sistemática formal, puesto que se recurre y muchos casos a la evaluación del "Mejor parecer", es decir, una evaluación subjetiva de criterio personal de valoración. Al respecto, Lafranceso V. (2003), sostiene que el problema fundamental de la Educación Superior Latinoamericana, es la "carencia de orientaciones teóricas educacionales que se advierte especialmente en la pedagogía. La importancia ha caído más en el empirismo. A los educadores se les critica por su interés en conocer hechos en lugar de encontrar el por qué (sic) de los mismos, y en el mejor de los casos, resolver los problemas que de estos hechos se describen, delimitan, definen y plantean" (p.19).

Como se ha observado, la formación profesional está basada en el desarrollo de los saberes profesionales: el saber conceptual o teórico, el saber procedimental o metodológico y el saber actitudinal o conductual. Esta adquisición progresiva de los saberes, deben ser evidenciados en un sistema de indicadores medibles; es decir, evaluables mediante procedimientos sistemáticos y con instrumentos válidos y confiables que permiten valorar objetivamente el estado cuantitativo y cualitativo de los aprendizajes.

En la actualidad la evaluación universitaria moderna se ha sistematizado, no solamente como el proceso que comprueba los aprendizajes y desarrollos obtenidos por los estudiantes y docentes, sino también, la evaluación como estrategia para lograr la calidad de la educación.: "la evaluación del sistema educativo contribuye a detectar sus fortalezas y 
dificultades, es por ello una estrategia de calidad. En la institución educativa la evaluación contribuye al mejoramiento de los recursos, actores, procesos y resultado"(Montenegro Aldana 2009). En lo pedagógico se asume la evaluación como como estrategia para alcanzar la calidad en los aprendizajes de los estudiantes. Por estas razones se hace necesario examinar el concepto de calidad educativa. En este aspecto, el concepto de calidad educativa, se ha anarquizado debido a la incursión de sistemas de calidad de la educación en los modelos neoliberales y el pragmatismo vigente en la concepción general de las acciones humanas en la educación y sus procesos.

La Calidad educativa, estando de acuerdo con Ignacio Abdón y Montenegro Aldana (2009), "en el contexto más amplio, es un indicador del desarrollo cultural de un pueblo o nación, es una característica que subyace en un sistema social económico y político. De acuerdo con la misión de Ciencia, Educación y Desarrollo la calidad de la educación está asociada a la "coherencia de todos los factores de un sistema educativo que colma las expectativas de una sociedad y, especialmente, de sus más altos críticos”.

Por otro lado, por la naturaleza de los datos que produce la evaluación como proceso, está en discusión la evaluación cuantitativa y cualitativa cuantitativa, cualitativa y también la combinación lógica cuantitativo - cualitativa lo que denominamos modelo mixto. Este último, en la práctica, con normas o sin ellas se aplica en todos los casos; por lo tanto, no constituye novedad o innovación significativa en el uso pedagógico de la evaluación de los aprendizajes fundamentalmente. Las categorías complejas de lo cuantitativo y cualitativo tienen fundamentación filosófica. Para la teoría dialéctica la cantidad y la calidad son unidades dialécticas inseparables; así, no hay cantidad sin calidad ni viceversa. Asimismo, para nosotros, no hay evaluación puramente cualitativa ni cuantitativa, todos los objetos, fenómenos y procesos de la realidad tienen necesariamente correlatos cuantitativos y cualitativos.

En la discusión entre la evaluación cualitativo - cuantitativa, en realidad lo que existe como en el caso de la investigación pedagógica, es la Evaluación predominantemente cuantitativa o predominantemente cualitativa, puesto que no existe una evaluación puramente cualitativa o evaluación enteramente cuantitativa; lo que existe, es una complementariedad dialéctica entre estas categorías medibles en los hechos; por lo que no existe cantidad separada de cualidad. En esencia ambas mediciones son necesariamente complementarias.

Como antecedente y origen del problema de la presente investigación, en la Educación Básica conformada por los niveles Educación Inicial, Primaria y Secundaria el sistema educativo peruano, ha venido aplicando la evaluación cuantitativa que expresa mejor la situación del fenómeno del aprendizaje; la forma pedagógica más sistematizada, objetiva y administrativamente más manejable puesto que allí interviene la Estadística Educativa, En este contexto surgen propuestas de evaluación cualitativa como modelos flexibles por su naturaleza subjetiva. En el trabajo docente puede resultar positiva si se maneja con cuidado, honestidad y ética profesional, sin embargo, tornarse en un facilismo académico de supuestos cálculos para satisfacer a los grupos interesados, quienes siempre esperan altas calificaciones como notas de sus hijos y ahora podrán posiblemente esperar "Logros Muy Satisfactorios". El sistema educativo peruano ha venido aplicando más el modelo cuantitativo $\mathrm{y}$, adicional, complementario y en casos excepcionales como en la Educación Inicial y Primaria, ensayando la Evaluación Cualitativa de expresión de calificativos en letras. Es lógico que en el nivel Inicial y Primaria puede ser aplicado la evaluación cualitativa y resulte adecuado y satisfactorio, sin embargo, no ser igual en aprendizajes complejos como las matemáticas y asignaturas forma- 
lizadas en la Educación Superior Universitaria. Lo que se considera ser eficiente y eficaz en este nivel es la evaluación cuantitativa.

Por otra parte, considerando que la evaluación actual es una estrategia de aprendizaje; es decir, la evaluación es un proceso inherente del aprendizaje. Por eso, nuestro supuesto es que, el Ministerio de Educación pretende aplicar esta evaluación subjetiva, para disimular el fracaso de la educación peruana que se expresa que el niño, el adolescente, en general todo aprendiz sometido a la evaluación cuantitativa, cada año y día tiene menos conocimientos en el cerebro y en las experiencias cognoscitivas. La unidad lógica entre la Teoría y la Práctica sobre el conocimiento que nos rodea ha disminuido constantemente. El conocimiento científico está reducido a un pretexto para desarrollar la competencia; esta se convierte en la unidad de medida del aprendizaje pragmático.

El Ministerio de Educación ha dispuesto sorpresivamente el uso de la evaluación cualitativa con calificativos con letras. Sin embargo, inmediatamente surgieron cuestionamientos de personalidades importantes como Idel Vexler, exministro de Educación reciente, sorprendido sostiene: "Es preocupante que de un momento a otro y faltando cerca de un mes para el inicio del año 2019 (sic) la viceministra pedagógica haya decidido generalizar en todos los colegios públicos y privados de Secundaria la evaluación cualitativa y usar los siguientes calificativos: LMS: LOGRO MUY SATISFACTORIO, antes AD, LS: LOGRO SATISFACTORIO, antes A; LB: LOGRO BÁSICO, antes B; LI: LOGRO INICIAL, antes C. Cuestionando metodológicamente, pregunta

1) ¿Por qué en vez de seguir usando la misma escala de letras de Primaria que ya conocen los maestros y los padres de familia, la combinación con nuevas letras?

2) ¿Por qué no se capacitó a los maestros?

3) ¿Por qué no se comienza con un "plan piloto" o con el primero de Secundaria?

Las observaciones del pedagogo, más que atinadas, son lógicas. Con la aplicación generalizada de la Evaluación cualitativa en Educación Secundaria, "El Perú es primero en la aplicación de la evaluación cualitativa utilizando letras. Es decir, un sistema educativo que está crisis permanente de sus resultados, resulta inadecuado la aplicación de sistemas más complejos y subjetivos. Se confirma que en el Currículo Nacional aprobado en 2016 se dispone con acierto extender ese sistema de evaluación a la secundaria" (Diario La República, sección Opinión, pág. 12 del viernes 1 de febrero de 2019).

La tendencia de la opinión pública en todos los medios impresos y las redes sociales masificadas es que, el sistema de evaluación, pronto se extenderá también a la Educación Superior Universitaria, así el Diario La República del día 30 de enero de 2019, p.19: Sociedad, da cuenta que "Minedu plantea cambiar los clásicos exámenes de admisión de universidades", lo cual busca que los postulantes ya no sean evaluados por sus conocimientos, sino por sus competencias; es decir, por sus saberes, capacidades y actitudes aplicadas a la resolución de problemas reales y a la vida diaria". Frente a esta propuesta el Rector de la Universidad Nacional Mayor de San Marcos "se mostró dispuesto a discutir la propuesta".

Una proyección de las tendencias muestra que la generalización de la Evaluación Cualitativa con calificativos expresados en letras podría extenderse pronto a la Educación Superior Universitaria. La Universidad es ahora, administrada por el Ministerio de Educación, por lo que es lógico suponer que la este proceso se extenderá a las universidades, puesto que ya está dispuesto y en aplicación en Educación Inicial, Primaria y Secundaria y luego pasaría 
a Educación Superior No Universitaria, la Educación Superior Universitaria y la Educación Superior de Posgrado.

En ese contexto arriba descrito, anticiparse a los hechos es función de la inteligencia humana y la ciencia, dada la premisa de la inminencia de la aplicación de la Evaluación cualitativa con expresión de calificativos en letras en las universidades. Con el propósito de una apertura de reflexiones preliminares sobre sus posibles implicancias en el sistema pedagógico y administrativo de la Universidad Nacional del Centro del Perú y con las premisas que anteceden se pretendido responder el problema general que estuvo expresado: ¿Cuáles serían las posibles implicancias pedagógicas y administrativas de la Evaluación Cualitativa con expresión de calificativos en letras en la Universidad Nacional del Centro del Perú? Los problemas específicos disgregados fueron:

1) ¿Existe necesidad real, pedagógica y administrativa para la aplicación de la Evaluación Cualitativa en el Sistema Universitario y, si existe, cuáles son los presupuestos y teóricos que sustentan tales necesidades?

2) ¿Cuáles serían las implicancias pedagógicas de la posible aplicación de la Evaluación Cualitativa con expresión de calificativos en letras en la Universidad Nacional del Centro del Perú?

3) ¿Cuáles son las implicancias administrativas de la posible aplicación de la Evaluación Cualitativa con expresión de calificativos en letras en la Universidad Nacional del Centro del Perú?

Coherente con los problemas, los objetivos formulados fueron los siguientes:

a) Objetivo general: Analizar las posibles implicancias pedagógicas y administrativas de la Evaluación Cualitativa con expresión de calificativos en letras en la Universidad Nacional del Centro del Perú.

b) Objetivos específicos:

1) Determinar las posibles implicancias pedagógicas de la aplicación de la Evaluación Cualitativa con expresión de calificativos en letras en la Universidad Nacional del Centro del Perú.

2) Determinar las posibles implicancias administrativas de la aplicación de la Evaluación Cualitativa con expresión de calificativos en letras en la Universidad Nacional del Centro del Perú.

3) Analizar las bases científicas de la necesidad real, pedagógica y administrativa para la aplicación de la Evaluación Cualitativa en el Sistema Universitario y, los presupuestos y teóricos que sustentan tales necesidades.

\section{Metodología}

El estudio se realizó en la Universidad Nacional del Centro del Perú, es de nivel descriptivo de prognosis exploratoria de tipo básica. El propósito es fundamentar si existe real necesidad estratégica, exigencias pedagógicas y administrativas que sustentan la necesidad de aplicar la Evaluación Cualitativa con calificativos expresados en letras simbolizadas en la Universidad Nacional del Centro del Perú (UNCP en adelante). 
La recopilación de datos se realizó mediante la técnica de la entrevista colectiva de Grupos focales con participación de 24 expertos considerados clave por las funciones de decisión que cumplen en la estructura administrativa de la UNCP. Las dimensiones de estudio son las implicancias pedagógicas e implicancias administrativas que podría ser afectadas en caso se aplicara el modelo de evaluación cualitativa con expresión de calificativos en letras.

Por la naturaleza de los datos que son discursos argumentados, en el procesamiento se aplicó las descripciones, interpretaciones y explicaciones argumentativas con con enfoque dialéctico de los fenómenos sociales, sin embargo, se adoptó la estructura de un artículo científico. La población focalizada, estuvo conformada por 24 expertos informantes elegidos por muestreo estratégico estratificado por conveniencia como: Tres de la alta dirección, seis decanos de facultades representativos de las áreas académicas estructuradas para los exámenes de admisión; seis Jefes de Departamento Académico de las respectivas facultades como del caso anterior, seis profesores pedagogos de la Facultad de Educación que desarrollan asignaturas de evaluación educativa; tres técnicos administrativos de la Oficina de Asuntos Académicos que tienen a su cargo el registro y control de las notas parciales y actas promocionales de las facultades y dos investigadores pedagogos especializados en elaboración de instrumentos de evaluación educativa. El muestreo estratégico se justifica, debido a la consideración funcional de las personas como elementos nucleares imprescindibles que por su ocupación, función, especialización o experiencia son portadores de datos y decisiones como unidades básicas de observación

La fuente de datos fueron los 24 informantes, en los que se utilizó tres guías de entrevista colectiva focalizada en los niveles estratégico o de alta dirección, el nivel gerencial que equivale a los departamentos académicos y el nivel operativo que constituyen los profesores y los técnicos administrativos. El material documental como fuente de datos fueron las normas más importantes como la Constitución Política de la República: Art. $18^{\circ}$ Fines de la educación universitaria: La educación universitaria tiene como fines la formación profesional, la difusión cultural, la creación intelectual y artística y la investigación científica y tecnológica. (...) La universidad es la comunidad de profesores alumnos y graduados. Cada universidad es autónoma en su régimen normativo, de gobierno, académico, administrativo y económico. Las universidades se rigen por sus propios estatutos en el marco de la Constitución y de las leyes. La Ley Universitaria No 20230 y sus reglamentos. Capítulo III Superintendencia Nacional de Educación Superior Universitaria (SUNEDU), Artículo $12^{\circ}$ al Artículo 29; el Estatuto de la Universidad Nacional del Centro del Perú, el Modelo pedagógico de la Universidad Nacional del Centro del Perú: Reglamento Académico General, de la UNCP; el Sistema de Gestión de las Organizaciones Educativas ISO 9001 de la facultad de Educación, el Sistema de Evaluación de la UNCP; el esquema y modelos de sílabos del desarrollo de asignaturas, los diseños curriculares y la Modelo de Acreditación para Programas de Estudios de Educación Superior Universitaria (SINEACE 2017).

En el procesamiento de datos, se recurrió a las estrategias de argumentación descriptiva, interpretativa y explicativa:

- El modelo descriptivo. Describe sistemáticamente las características y las condiciones en que se dan las variables de estudio, a fin de identificar la naturaleza de los fenómenos investigados; limitándose a la posición discursiva de los informantes.

- Deductivo interpretativo. Se orienta a acumular evidencias, compatibilidades y contradicciones, inconsistencias y limitaciones; busca interpretar las causas de los fenómenos. 
Aplica argumentos, hermenéuticos, estadísticos, causales y simbólicos. Se inicia con la exposición de los conceptos, categorías, leyes y teorías que sustentan su investigación. Sube y baja sus explicaciones desde niveles concretos a niveles abstractos de la realidad y sus generalizaciones. Es el trabajo que necesita alta complejidad del pensamiento analítico, crítico, creador e innovador. Utiliza datos y hechos, argumentos y generalizaciones coincidentes, coherentes con las leyes del desarrollo de la realidad como propiedad de la ciencia.

- Inductivo explicativo. El modelo combina la práctica directa del trabajo con las teorías sustentadas. Establece las relaciones lógicas entre las prácticas y los discursos de los informantes, así como caracteriza a los objetos y fenómenos de la investigación en sus correspondientes unidades de análisis y de observación. Sirve para desarrollar y construir la parte teórica de la investigación cualitativa. Deduce, interpreta e intenta explicar el porqué de los fenómenos. generalizar. Igualmente se ha recurrido al uso de la hermenéutica para interpretar los datos formales simbolizados.

El análisis dialéctico implicó la consideración de los principios de la sociología dialéctica materialista de los fenómenos sociales que fundamenta la interdependencia de las relaciones económico - sociales sobre la superestructura social del hombre y la sociedad. Considera a los fenómenos sociales, altamente dialécticas, contradictorias y en pugna constante entre lo nuevo y lo viejo, lo cualitativo y lo cuantitativo, lo concreto y lo abstracto, etc.

Las estrategias seguidas fueron:

- Los expertos recibieron una guía de entrevista con el temario y los procedimientos de la entrevista colectiva.

- Los expertos considerados para cada grupo focal, expresaron sus opiniones, pareceres, apreciaciones y criterios respecto a la supuesta evaluación cualitativa con calificativos en letras que podrían ser implementadas en la Universidad Nacional del Centro del Perú.

- La conducción posible implementación del Ministerio de Educación, la Evaluación Cualitativa con expresión de calificativos en letras. Para poder continuar con el desarrollo de nuestra investigación y la consecución de los objetivos propuestos en el proyecto, se debe viabilizar la ejecución de la recopilación de datos que nos permita el análisis, síntesis y su posterior inferencia de posibles implicancias pedagógicas y administrativas de la evaluación cualitativa y posterior.

Los datos recopilados se organizaron resúmenes de extractos en una Matriz de datos cualitativos. Por sus propósitos de investigación exploratoria, se ha determinado los ejes temáticos para el análisis mediante la construcción de un esquema gráfico de flujos. Se optó por la metodología cualitativa de recogida y el análisis por descripción, interpretación y explicación, porque los datos en esta técnica son discursos orales donde puede captar las concepciones y visión del mundo de la ciencia, la educación y otros procesos sociales como la educación y sus medios.

\section{Resultados}

Los datos obtenidos mediante procesos metodológicos expuestos intentaron responder el problema principal: ¿Cuáles serían las implicancias pedagógicas y académico-administrati- 
vas de la Evaluación Cualitativa con expresión de calificativos en letras, si se aplicara en la UNCP? Coherente con la estructura del problema, los objetivos específicos que orientaron la investigación son:

1) Determinar las implicancias pedagógicas de la posible aplicación de la Evaluación Cualitativa con expresión de calificativos en letras.

2) Analizar las implicancias académico administrativas de la posible aplicación de la Evaluación Cualitativa con expresión de calificativos en letras.

3) Sistematizar las bases científicas de la necesidad real, pedagógica y administrativa para la aplicación de la Evaluación Cualitativa en el Sistema Universitario y, los presupuestos y teóricos que sustentan tales necesidades.

Con las premisas que anteceden en resumen, los resultados de la investigación son:

\section{Constructos elaborados}

Implicancias. El término implicancias es polisémico. Para la presente investigación se ha definido como: consecuencias, secuelas, contradicciones o incompatibilidades de la evaluación cualitativa cuyos calificativos son expresadas en letras simbolizadas, con respecto a los calificativos con expresión numérico cuantitativos con uso de los medios matemático estadísticas.

Evaluación. Estando de acuerdo con Ya Tyler (1950), la evaluación es «El proceso esencialmente de determinar, hasta qué punto los objetivos educativos han sido actualmente alcanzados mediante los programas y currículos de enseñanza».

Naturaleza de la evaluación. La evaluación es un proceso complejo de medición y valoración de logros planificados en la enseñanza. No es fácil, ni necesario tener un concepto único para la evaluación. Resulta absurdo buscar a tener un concepto único de evaluación de aprendizajes, pues, así como existe una gran diversidad de aprendizajes, debe haber también diversidad de evaluaciones La Evaluación es una estrategia de aprendizaje que consiste en valorar o estimar lo logrado por el estudiante en función de lo planeado por el profesor. Proporciona información para reorientar las prácticas pedagógicas. (Montenegro Aldana Ignacio (2009).

Calificativos de evaluación. Sistema de asignación de valores numéricos, símbolos, códigos o categorías expresadas en abreviaturas en letras. "La calificación es la asignación de un sistema de valores que expresan la escala de puntuaciones de un sistema de calificaciones" (Montenegro (2007).

Evaluación cuantitativa. Evaluación con instrumentos válidos y confiables cuya expresión de calificativos son numéricas. Permite procesar estadísticamente, su publicación y archivamiento sistemático.

Evaluación cualitativa. Procesos valorativos que consideran también los hechos subjetivos, las apreciaciones personales y derivan en opiniones y argumentos que pueden convertirse en hechos políticos puesto que es un proceso de juzgamiento desde el punto de vista del evaluador.

Calificación en letras. Son la expresión de categorías simbólicas en letras que necesariamente tienen correlatos cuantitativos. Sus argumentos son una influencia del psicologismo esotérico que sostiene que los bajos calificativos generan bajo autoestima de los estudiantes. 
Evaluación de la calidad y acreditación. Conjunto de procedimientos y acciones normadas (reglamentadas) que permiten recoger información pertinente, procesarlas, valorarlas y tomar decisiones sobre la calidad, cantidad y operatividad de aprendizajes incorporados al saber, saber hacer y saber profesional de los estudiantes (SINEACE: Modelo de Calidad para la Acreditación de Carreras de Educación).

Evaluación de entrada. Denominada también diagnóstica. Permite al docente, establecer el perfil de los conocimientos previos del estudiante para iniciar nuevos aprendizajes a través de las asignaturas.

Evaluación de proceso o formativa. Es la evaluación más importante procesada por el profesor en las acciones pedagógicas de la enseñanza - aprendizaje. Se utiliza como estrategia motivadora, un medio de establecer diálogos académicos; actúa como rompehielos comunicativos, es fundamentalmente un sistema para consolidar saberes y experiencias incorporadas en los estudiantes. Es un proceso de reforzamiento operativo que tiene como propósito, consolidar los procesos educativos en su fase de desarrollo.

Evaluación de salida. Denominada también sumativa. Evaluación que se propone contrastar la eficacia general del proceso educativo ya desarrollado, esto es, la eficacia de los resultados del aprendizaje o la eficacia general de un programa educativo.

Evaluación del perfil de egreso. Es evaluación de final de carrera o evaluación de control de perfil de egreso en las competencias programadas del diseño curricular del programa de estudios. Es una evaluación que controla el logro o no conformidad de la competencia general desarrollada a través de las asignaturas, expresadas en el perfil de egreso.

Articulación enseñanza, aprendizaje y evaluación. Unidad de procesos de interactividad, interdependencia y de complementariedad entre la enseñanza-aprendizaje y la evaluación. Se evalúaloquese enseñayloque el estudiantehaaprendidopormediodela enseñanzadelmaestro.

Evaluación integral. Evaluación que considera todos los aspectos de la dimensión humana individual y social: entradas, procesos y salidas del desarrollo general del hombre y su integración productiva a la sociedad y la comunidad.

Articulación evaluación, información y comunicación. Proceso por el cual los elementos del aprendizaje, la comunicación, la información y la evaluación se desarrollan concatenados para cumplir adecuadamente la formación docente.

\section{Ejes temáticos de la investigación}

Las categorías, llamadas también ejes temáticos sobre los cuales giran los datos y evidencias empíricas, así como los distintos productos empíricos obtenidos en nuestras observaciones como participantes directos e investigadores participantes de los procesos estudiados, se tratado tres ejes temáticos:

- Implicancias pedagógicas de la evaluación cualitativa

- Implicancias académico administrativas de la evaluación cualitativa

- Necesidad real de la evaluación cualitativa

\section{Implicancias pedagógicas}

La educación formal es el ejercicio profesional de los maestros cuya ciencia vertebral de su formación es la Pedagogía. Esta, estudia el fenómeno social de la educación que consiste 
en la formación integral del hombre en sus dimensiones física, psíquica y sociocultural, preparándolas para su participación productiva, ética y responsable en la sociedad en que vive. Sin embargo, en las universidades con distintas facultades y carreras profesionales, la planificación, implementación, ejecución de la enseñanza - aprendizaje y la evaluación es realizada por profesionales tanto pedagogos y profesionales no pedagogos. De ahí, las percepciones de la evaluación que nos ocupa ahora, no solamente son distintas, sino, confusas, contradictorias hasta antipedagógicas.

En el contexto descrito, las Implicancias pedagógicas de una evaluación cualitativa con calificativos en letras de códigos simbolizados, está referido a las probables consecuencias, secuelas, contradicciones o incompatibilidades que produciría la aplicación de la evaluación cualitativa con calificativos en letras, de ser aplicadas en los procesos pedagógicos de la evaluación de los aprendizajes, el promedio ponderado, las estadísticas educativas de promoción y ubicación de los estudiantes según niveles de rendimiento académico y los méritos académicos del desarrollo de las competencias de la UNCP, probablemente tendría graves dificultades por estar habituados en la evaluación cuantitativa del modelo matemático - estadístico que ha resultado, adecuada, objetiva, válida y confiable hasta ahora. Es así que el sistema educativo peruano, en particular el universitario, aplica desde sus orígenes como educación superior universitaria formal, el modelo cuantitativo matemático - estadístico que ha facilitado la sistematización, archivamiento y seguridad de los datos, resultados de la formación profesional universitaria.

En este eje temático de la investigación se ha obtenido datos a partir de una muestra estructurada intencional, estratégico, mediante entrevistas colectivas en los grupos focales que conforman la Alta Dirección, decanos, directores de departamentos académicos, profesionales de la educación que desarrollan asignaturas de evaluación educativa. Sin embargo, precisamos que la unidad de análisis Alta Dirección se debe considera como mortalidad muestral, puesto que no se ha podido cumplir con la entrevista focal, debido a la situación de emergencia sanitaria por la pandemia del COVID 19 que generó el aislamiento social y cuarentena, pasando la gestión académica y gestión institucional, de la modalidad presencial a la modalidad no presencial, virtual o remota.

Por otra parte, para el análisis de las Implicancias pedagógicas, es necesario sustentar el rol de la evaluación en la formación por competencias. Esta implica, la evaluación de habilidades complejas tanto conceptuales, procedimentales y actitudinales. Además considerar las prácticas evaluativas de los docentes pedagogos y de otras carreras profesionales; así como es necesario evaluar qué hacen los estudiantes con los conocimientos y saberes que adquieren en diferentes contextos. La evaluación moderna tiene también como función, orientar, estimular y proporcionar información y herramientas para que los estudiantes progresen en su aprendizaje.

Para la sistematización de los datos obtenidos que son productos empíricos, se ha diseñado un mapa de flujos que refleja la secuencia del análisis de datos. 


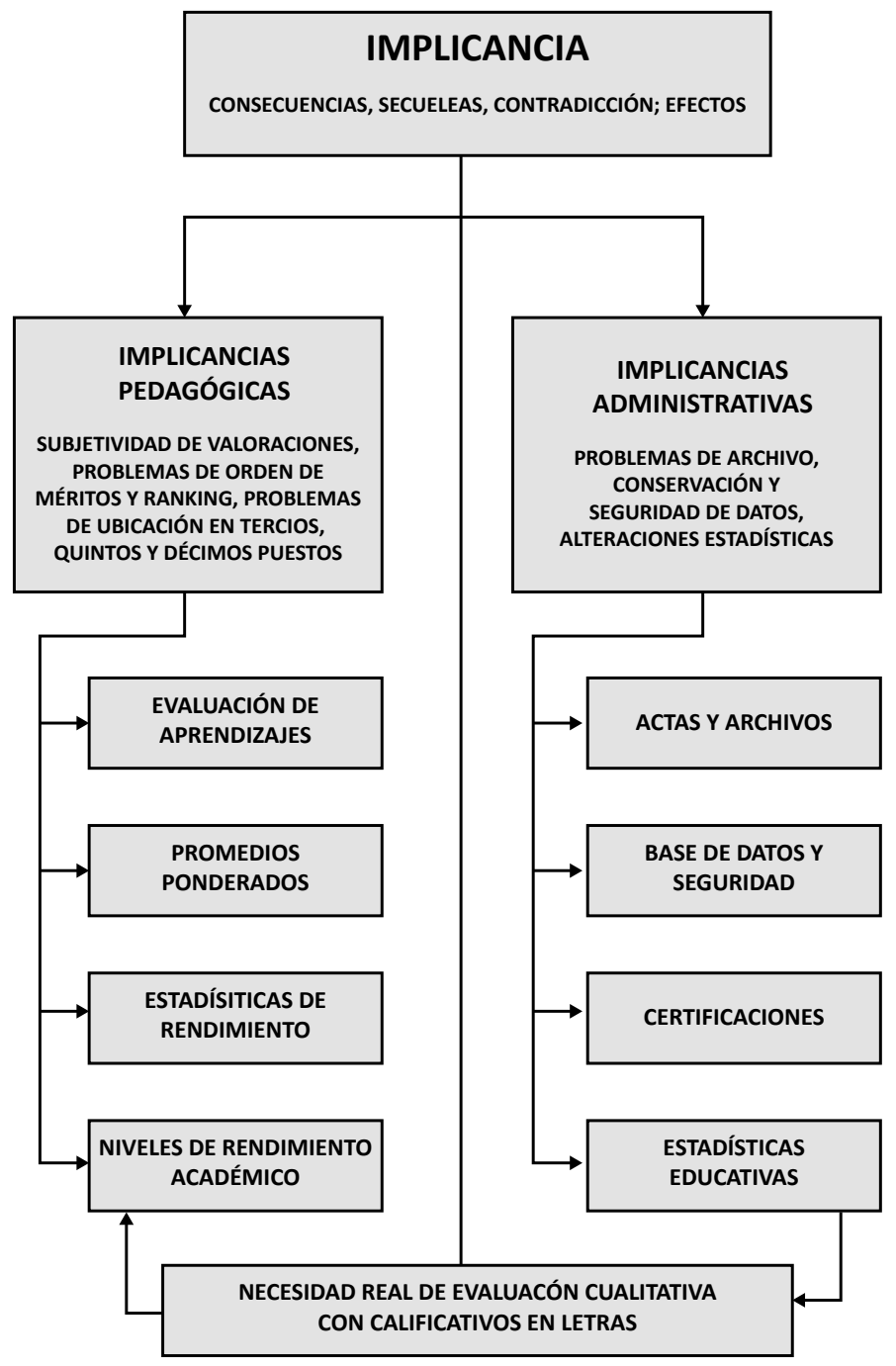

En el eje de Implicancias pedagógicas se ha identificado cuatro grupos de opinión:

Los legalistas. Manifiestan en general que aceptarían la evaluación cualitativa como innovación educativa, siempre en cuando esté legislada y consensuada. Es el grupo mayoritario de las opiniones, predominantemente conformados por las carreras de áreas técnicas, quienes se resisten a reflexionar o discutir de aquello que no existe aún, como la Evaluación Cualitativa con expresión de calificativos en letras justificándolo es potencial, Sus argumentos basan en la ausencia de bases legales y normativas de un sistema cualitativo no implementado. Sin 
embargo, este grupo, no visualiza la función de las investigaciones exploratorias, de prognosis o predictivas que tiene la ciencia, de anticiparse al suceso de los fenómenos, previo conocimiento de las leyes del desarrollo de los fenómenos tanto de la naturaleza, la sociedad y el pensamiento. El informante D04, sostiene que "La Ley Universitaria no contempla este tipo de evaluación. NO TENDRÍA UN VALOR LEGAL por considera que no se ubica en el futuro de los fenómenos que podrían ocurrir.

Los negacionistas. Afirman desconocer la evaluación cualitativa en la Universidad y se resisten en la posibilidad de la evaluación cualitativa en la Universidad. El argumento de no tener información sobre el hecho supuestamente justifica no abordar la evaluación cualitativa en la Universidad; por lo que "no se puede hablar de algo que no existe y no está implementado". Si se diera el caso, se debe "buscar consenso y capacitación”. Implícitamente están de acuerdo con los cambios, sin embargo, los tres docentes no precisan las implicancias que podrían afectar o favorecer a la formación profesional y la administración de sus datos de la evaluación. No es necesario otro tipo de evaluación.

Los agnósticos. Sostienen que la evaluación no es problema en la Universidad. La evaluación puede ser cuantitativa o cualitativa y no incide en la formación profesional. Lo que se debe cuidar es que los conocimientos que adquieren debe ser útiles para su carrera profesional y su vida práctica. Al parecer es el grupo pragmático utilitarista.

Los que abogan por la no contradicción de lo cuantitativo y cualitativo. Sostienen con diferentes matices, que la evaluación en sus modalidades cuantitativas y cualitativas, no constituyen problemas y tienen una relación de complementariedad análoga a las investigaciones de estos tipos. Seis informantes focales conforman este grupo que relativizan las contradicciones que podrían implicar si se diera el caso. "No existe contradicción entre lo cualitativo y lo cuantitativo, lo que hay, es complementariedad". El grupo es conformado por profesores pedagogos de carrea, probablemente influenciados por la filosofía cuya disciplina es obligatoria en su formación. No cabe nuevas discusiones sobre lo que existe lo que dialécticamente coexisten.

\section{Implicancias académico administrativas}

Responde al problema específico, ¿Cuáles serían las Implicancias Administrativas de la Evaluación Cualitativa con expresión de calificativos en letras, si se diera el caso en la $U N C P$ ? En este eje temático el análisis comprende los problemas de archivo, custodia y seguridad de datos de la evaluación. Están categorizados en los procesos fundamentales de actas y archivos, base de datos y seguridad, certificaciones y estadísticas educativas. Aquí encontramos que, un solo informante (D04) y cinco integrantes del grupo focal, conocen los procesos de la evaluación cualitativa, sin embargo, muestran limitaciones conceptuales $\mathrm{y}$ de alcances de la evaluación en lo administrativo: Al inquirirnos "iQué tiene que ver la evaluación con lo administrativo!", reflejan tácitamente sobre las implicancias de la evaluación en lo académico administrativo. No reconocer o admitir las implicancias en lo administrativo, se desconoce que la evaluación se concibe como proceso y como producto. Como proceso, la actividad por el cual se planifica la evaluación, se construye instrumentos válidos y confiables; recoge información confiable sobre el aprendizaje de los estudiantes, se valora mediante una nota que puede ser numérica o cualitativa en letras, palabras o frases que tiene un correlato cuantitativo de intervalos; se comunica los resultados y 
se toma decisiones correctivas, reforzamiento o retroalimentación. Como producto es la información que pasa a los administrativos, quiénes elaboran estadísticas de promedios ponderados, cuadro de méritos, clasificación de estudiantes por el nivel de aprovechamientos como tercios, quintos y décimos; se hacen certificaciones y luego se pone en custodia y seguridad de la información.

\section{La necesidad real de la real de la evaluación cualitativa}

El problema formulado para este eje es ¿Existe necesidad real, estratégica o administrativa para la aplicación de la Evaluación Cualitativa en el Sistema Universitario en la UNCP? Los informantes decanos (D01 y D03) de dos facultades técnicas, mencionan que "el sistema de evaluación cualitativo es necesario" argumentando las nuevas formas de enseñanza aprendizaje. Otro Decano muestra con algo de apertura, sin embargo al igual que los otros tres sostienen que no hay necesidad en el momento. Lo que se interpreta, que si se diera el caso, aceptarían.

Estando de acuerdo con Blanco A. (2016), planteamos una evaluación integral, teniendo como requisito, una enseñanza científica del mismo carácter. "Los cambios demandan un modelo de educación centrado en el aprendizaje y en la formación integral de los estudiantes, lo que implica un cambio de rol tanto para el docente como para el alumno, con el consiguiente replanteamiento de las competencias en Educación Superior. Los autores aportan una definición, desarrollo y evaluación de competencias genéricas que se pretenden desarrollar en el estudiante universitario. Se proponen como competencias clave, la planificación de objetivos, la responsabilidad, el pensamiento crítico, el trabajo en equipo, la comunicación oral, la creatividad y la innovación, la comprensión interpersonal y las habilidades comunicativas, así como, el uso de las TIC en la búsqueda y elaboración de la información, el manejo de la interculturalidad en el aula, la negociación y el manejo de conflictos. (p.14).

\section{Discusión}

Metodológicamente, la presente investigación, por su carácter de prognosis predictivo en las variables pedagógica, administrativa y de necesidad real, está contextualizado en el modelo cualitativo predominantemente interpretativo del discurso informativo de los participantes del grupo focal. El aspecto predictivo está basado en el carácter exploratorio predictivo de la ciencia, por el cual, el conocimiento científico en su proceso, la investigación científica tiene la capacidad de predecir, tanto fenómenos naturales, sociales y del pensamiento, debido al conocimiento de las leyes del desarrollo del fenómeno investigado. Por otra parte, es también necesario prever las probabilidades de ocurrencia de otros fenómenos contrarios al avance de la calidad de la formación universitaria.

En síntesis y en consecuencia de las normas existentes, la universidad tiene cuatro grandes parámetros de la evaluación que determinan la calidad de la universidad: 1) la evaluación de los aprendizajes de los estudiantes como productos de la enseñanza del docente, 2) la evaluación de la Investigación o el conocimiento producido por docentes y estudiantes, 3) evaluación de la Docencia; es decir, el desempeño profesional docente y 4) evaluación de la Gestión y sus resultados. De ahí que, la universidad universitaria es permanente y "siempre 
evalúa a estudiantes, docentes, trabajadores; evalúa productos como la investigación, la proyección, así también los desempeños docentes" (Tarazona 2016).

Los resultados de la investigación, procesado ya, las respuestas emitidas por los integrantes de los grupos focales en líneas generales son superficiales, ambiguas y de un nivel incipiente de informaciones sobre los tipos de evaluación que existe en la Universidad tanto en la variable pedagógico - académico administrativo y en las explicaciones de la necesidad real de la Evaluación cualitativa con calificativos en letras.

Teniendo en cuenta que la presente investigación tiene carácter exploratorio, de intención predictiva que ha tenido limitaciones significativas en el acceso a informaciones más sistematizadas, debido a la sitación de emergencia sanitaria de aislamiento social, exige una réplica en situaciones normales de enseñanza y aprendizaje presenciales a lo que denominamos normales. La entrevista grupal con participantes a los que hemos denominado informantes focalizados $\mathrm{y}$, dada las condiciones imprevistas de la situación de emergencia sanitaria y de aislamiento social obligatorio, ha sido un factor limitante para el levantamiento de datos empíricos suficientes. Sin embargo, se ha recurrido a otras estrategias alternativas, según permite la metodología cualitativa, recurrir a la triangulación metodológica y la especulación teórica.

El propósito de la investigación se ha logrado en una parcialidad aproximada del $80 \%$ en acopiar opiniones experimentadas de los gestores, quienes están en ejercicio de las funciones de gestión actual como personal académico (docentes) y personal operativo (administrativos) cuya práctica ocupacional funcional, involucra la evaluación en su diversidad de formas existentes en la organización, que necesariamente implica la obtención de puntuaciones que deben ser expresados numérica o literalmente categorizados o simbólicos.

La información recogida en grupos focales y analizada mediante la argumentación descriptiva, interpretativa y explicativa deductiva ha sido interpretado en el contexto de la pedagogía universitaria y los términos de la administración, gestión o gerencia basados en el enfoque sociocrítico, la sociología dialéctica, la especulación conceptual, tratando de alguna manera de desprendernos de los paradigmas conocidos y muchas veces, puestos en moda en las indagaciones y exigidos institucionalmente. La investigación basada en la concepción neoliberal de Investigación, desarrollo e innovación ( $\mathrm{I}+\mathrm{D}+\mathrm{i})$, cuyo producto expresa en el modelo restringido de un artículo científico trabajado fundamentalmente en modelos exclusivos dominantes de las revistas científicas, que ata a la investigación y el investigador en un esquema mental del pragmatismo que anula la flexibilidad, diversidad metodológica, la imaginación, creación y adecuación a contextos de la realidad. En ese contexto, los resultados más importantes de la investigación son los que estuvieron previstos en los objetivos:

La hipótesis general, es apoyada por los datos empíricos. La proposición: La Evaluación Cualitativa con calificativos expresados en letras tendría implicancias negativas en la gestión pedagógica y académico administrativa de la Universidad Nacional del Centro del Perú, en cuanto, por lo menos en los inicias de su aplicación, se constituiría en un factor que anarquiza la medición y la cuantificación de los productos laborales del docente, el aprendizaje de los elementos conceptuales, procedimentales y actitudinales de los estudiantes y como consecuencias administrativas, rompería el esquema formalizado del sistema de almacenamiento de información con técnicas matemático estadísticas y no permitiría la jerarquización sistemática.

Respecto a las hipótesis específicas, las informaciones obtenidas apoyan el sentido general de las hipótesis formuladas en el Plan de investigación. 
1) Se advierte que no existe necesidad real, pedagógica ni administrativa para la aplicación de la Evaluación Cualitativa en el Sistema Universitario, puesto que sus presupuestos teóricos están basados en el psicologismo esotérico y los actuales sistemas de archivo están estructurados en los principios de la informática basada en la estadística como ciencia formal.

2) Las posibles implicancias pedagógicas de la aplicación de la Evaluación Cualitativa con expresión de calificativos en letras en la Universidad Nacional del Centro del Perú se manifestarían en una anarquía epistemológica y metodológica de la formación por competencias.

3) Las posibles implicancias administrativas de la aplicación de la Evaluación Cualitativa con expresión de calificativos en letras en la Universidad Nacional del Centro del Perú, crearía potencialmente dificultades en el procesamiento de datos estadísticos y su comunicación al usuario y los procesos administrativos de formulación estadística y desnaturalizaría los sistemas formalizados que se tiene como archivos jerarquizados, las certificaciones serían caóticas y la ubicación de los estudiantes por el nivel de sus rendimientos académicos, serían cuestionables.

\section{Conclusiones}

Los datos encontrados nos permiten arribar a las siguientes conclusiones

No existe necesidad prioritaria pedagógica ni administrativa para implementar el uso de la evaluación cualitativa con expresión de calificativos en letras en la evaluación del aprendizaje ni en los procesos académicos y de gestión.

Las posibles consecuencias de la aplicación de la evaluación cualitativa con expresión de calificativos en letras generaría una anarquizaría de los datos estadísticos y dificultaría no solo el manejo de datos, sino también el sistema de archivos que no ayudaría la toma de decisiones por su naturaleza subjetiva.

Se deduce que, en el contexto de la interpretación dialéctica de los fenómenos sociales, existe una relación de complementariedad simultánea y viceversa entre la evaluación cuantitativa y cualitativa, análoga a las relaciones entre las investigaciones cuantitativas y cualitativas que son dialécticamente complementarias, puesto que no hay cantidad y calidad en toda materia ante la valoración de los sujetos de valor.

Se ha encontrado que existe una noción difusa y superficial de los informantes respecto a la evaluación cualitativa con expresión de calificativos en letras.

La posible aplicación de la evaluación cualitativa con expresión de calificativos en letras, según apreciaciones de los informantes de los grupos focales expertos abordados, constituyen una forma de desnaturalizar la medición válida y confiable mediante procedimientos de la estadística como herramientas del procesamiento y almacenamiento de datos de la educación y la gestión administrativa. 


\section{Referencias}

Batalloso N., Juan M. (2006). La Educación como responsabilidad social: Bases para un nuevo paradigma educativo. San Marcos, Lima, Perú.

Blanco, Ascensión (2016). Desarrollo y competencias en Educación Superior. Narcea Ediciones; Buenos Aires, Argentina.

Fernández M., Ampro (2014). La evaluación de los aprendizajes en la universidad: nuevo enfoque. Instituto de Ciencias de la Educación; Universidad Politécnica de Valencia, España.

García R., José M. Bases pedagógicas de la evaluación: Guía práctica para educadores. Síntesis, Madrid, España.

Hallak, Jacques. Educación y globalización. Libro electrónico en Internet.

Ibarrola N., María (2018).Temas claves de la evaluación de la educación básica. Fondo de cultura económica; México.

Lafrancesco V., Giovanni M. (2003). La investigación en Educación y Pedagogía: fundamentos y técnicas. Magisterio, Bogotá, Colombia.

Perú, Ministerio de Educación (2002). Programa de formación continua de docentes en servicio en Educación Secundaria. Lima, Perú.

Perú, Ministerio de Educación (2009).Diseño Curricular Nacional de Educación Básica Regular: Proceso de articulación. MED. Lima, Perú.

Perú, Ministerio de Educación (2016). Currículo Nacional de la Educación Básica: Programa Curricular de Educación Secundaria: MED; Lima, Perú.

Montenegro A. Ignacio A. (2009). Cómo evaluar el aprendizaje escolar. Magisterio; Bogotá, Colombia.

Montenegro A., Ignacio A. (2009). Evaluación del desempeño docente: Fundamentos, modelos e instrumentos. Magisterio, Bogotá, Colombia.

Morán O. Porfirio (2012). La evaluación cualitativa en los procesos y prácticas del trabajo en el aula (PDF) Instituto de Investigación Universidad y Educación; México.

Morán O., Porfirio (2012). Hacia una evaluación cualitativa en el aula. PDF, disponible en http:// www.iisue.unam.mx Universidad Nacional Autónoma de México: Instituto de Investigaciones sobre la Universidad y la Educación; México.

Santos G., Miguel A. 1995. En: La evaluación cualitativa en los procesos y prácticas en el aula de Porfirio Morán Oviedo. 2012, México.

Sevilla E., Julio C. (2003). Alumnos y docentes investigadores creativos. En: Umbral: Revista de Educación, Cultura y Sociedad UNPRG; Lambayeque, Perú.

Tarazona P. Filomeno (2016). Planeamiento Estratégico Aplicado a la Educación. Marsant's; Huancayo, Perú.

Tyler, ya; 1950, Citado por Mora V. Ana I. (2004).La Evaluación Educativa: conceptos, períodos y modelos. En: Revista Electrónica “Actualidades Investigativas en Educación”, vol. 4, núm. 2 julio - diciembre, 2004.

Toapanta J., Gladys L. (2016). "La evaluación cualitativa y la destreza oral del idioma inglés en los estudiantes del segundo año de Bachillerato General Unificado de la unidad educativa "Salcedo". Universidad Técnica de Ambato, facultad de Ciencias Humanas y de Educación, carrera de Idiomas; Ambato, Ecuador.

Universidad Nacional del Centro del Perú (2016). Estatuto de la UNCP; Huancayo, Perú.

Universidad Nacional del Centro del Perú (2016). Modelo Educativo; Huancayo, Perú.

Universidad Nacional del Centro del Perú (2019). Reglamento Académico General (versión 2) UNCP, Huancayo, Perú. 